\title{
DISTRIBUIÇÃO DE Hg TOTAL E SUAS ASSOCIAÇÕES COM DIFERENTES SUPORTES GEOQUÍMICOS EM SEDIMENTOS MARINHOS DA MARGEM CONTINENTAL BRASILEIRA: BACIA DE CAMPOS - RIO DE JANEIRO
}

Beatriz Ferreira Araujo*, Marcelo Gomes de Almeida, Marcos Sarmet Moreira Barros Salomão, Renato Rosa Gobo, Verônica Caldas Siqueira, Álvaro Ramon Coelho Ovalle e Carlos Eduardo de Rezende

Laboratório de Ciências Ambientais, Centro de Biociências e Biotecnologia, Universidade Estadual do Norte Fluminense, 28013-600 Campos dos Goytacazes - RJ, Brasil

Recebido em 14/10/08; aceito em 1/10/09; publicado na web em 11/3/10

\begin{abstract}
TOTAL MERCURY DISTRIBUTION AND ITS ASSOCIATION WITH DIFERENT GEOCHEMICAL SUPPORTS IN MARINE SEDIMENT FROM THE BRAZILIAN CONTINENTAL MARGIN: CAMPOS BASIN. Mercury distribution and geochemical support on the Continental Margin was evaluated at the Campos Basin, Rio de Janeiro state, Brazil. The average concentrations for all analyzed elements were, respectively, $20 \pm 5 \mathrm{ng} \mathrm{g}^{-1}(\mathrm{Hg}) ; 30 \pm 14 \mathrm{mg} \mathrm{g}^{-1}(\mathrm{Al}) ; 16 \pm 6 \mathrm{mg} \mathrm{g}^{-1}$ (Fe), and $254 \pm 83 \mu \mathrm{g} \mathrm{g}{ }^{-1}(\mathrm{Mn})$. Silt and clay content, total carbonate and $\mathrm{Hg}$, and organic carbon increased with depth. Finally, the relationship between $\mathrm{Hg}$ and silt clay showed significant positive correlation. Total $\mathrm{Hg}$ concentrations are the background level described primarily $\left(\sim 40 \mathrm{ng} \mathrm{g}^{-1}\right)$.
\end{abstract}

Keywords: mercury; Continental Margin; carbonate.

\section{INTRODUÇÃO}

O mercúrio é um metal que está presente naturalmente em baixas concentrações nos oceanos, tendo seu aporte no ambiente por uma variedade de processos naturais (ex. erupções vulcânicas) e antrópicos (ex. atividades industriais e agrícolas). Este elemento é reconhecido como um dos metais com maior potencial tóxico, por ser capaz de bioacumular nos organismos e biomagnificar através da cadeia alimentar. ${ }^{1}$

Os sedimentos atuam como importantes reservatórios de metais, ${ }^{2}$ sendo intensamente utilizados em diferentes tipos de abordagens científicas assim como em programas de monitoramento ambiental. Devido as suas características integradoras, este compartimento é um importante indicador sobre processos que ocorrem tanto nos sedimentos quanto na coluna d'água, podendo trazer informações a respeito da origem do material depositado, ou seja, de origem litogênica ou autigênica. ${ }^{3}$

O mercúrio apresenta baixa solubilidade na coluna d'água e o uso do coeficiente de partição geoquímico tem demonstrado que o material particulado em suspensão é responsável pelo transporte desse elemento na interface entre os ecossistemas continental e marinho, mesmo com os elevados fatores de diluição. ${ }^{4,5}$ Nos sedimentos de fundo este elemento estará associado às partículas e seus suportes geoquímicos orgânicos e inorgânicos (ex. carbonatos, óxidos e hidróxidos de $\mathrm{Al}, \mathrm{Fe}$ e Mn, matéria orgânica e sulfetos) assim como na água intersticial (ex. matéria orgânica dissolvida e/ou coloidal, fosfatos). ${ }^{6,7}$ A biodisponibilidade e toxicidade do $\mathrm{Hg}$ neste compartimento pode ser afetada por modificações pós-deposicionais que alteram parâmetros, como concentração de oxigênio dissolvido, potencial redox, $\mathrm{pH}$ e temperatura da água intersticial, gerando remobilização de $\mathrm{Hg}^{.}{ }^{8}$

No ambiente oceânico, na interface entre a plataforma e a margem continental e no ambiente profundo, a relação entre fontes e imobilização de material particulado em suspensão e suas associações orgânicas e inorgânicas possui muitas incertezas, principalmente em relação aos mecanismos de preservação e transformação de matéria orgânica, nutrientes e metais nos sedimentos de fundo. Os resultados obtidos em estudos anteriores de ambientes marinhos sobre o transporte e a

*e-mail: bfaraujo@yahoo.com.br deposição de material na interface continente-oceano compartimentam as seguintes regiões, a saber: $\sim 4 \%$ retidos em ambientes deltáicos; $\sim 45 \%$ retidos entre a plataforma e a margem continental; $\sim 6 \%$ retidos em zonas de alta produtividade (ex. ressurgências); 5\% nas regiões pelágicas de oceano profundo. ${ }^{9} \mathrm{~A}$ contaminação deste compartimento pode apresentar efeitos adversos nos ecossistemas aquáticos através do impacto nas estruturas das comunidades bentônicas, ${ }^{10,11}$ possibilitando a transferência do Hg através da cadeia trófica e sua biomagnificação.

Outro aspecto relevante é o funcionamento da compartimentação descrita acima em rios de médio porte, como é o caso da região que vem sendo estudada por nosso grupo. No caso da Bacia de Campos, litoral norte do Estado do Rio de Janeiro, existem duas principais entradas de mercúrio. A fonte difusa é representada pelas atividades de exploração e produção de petróleo que são capazes de introduzir no meio marinho efluentes enriquecidos em substâncias orgânicas e inorgânicas, ${ }^{12}$ entre elas o mercúrio, uma vez que este elemento tem sido detectado na composição das águas de produção. ${ }^{3}$

A fonte pontual é representada pelo rio Paraíba do Sul, o qual tem sido estudado ao longo das duas últimas décadas principalmente devido ao uso do $\mathrm{Hg}$ nas plantações de cana-de-açúcar da região, através de fungicidas organomercuriais e pela atividade de garimpo. ${ }^{13}$ Estes estudos anteriores mostraram um nítido enriquecimento dos sedimentos superficiais sob influência da pluma fluvial. Em adição, a determinação do $\mathrm{Hg}$ em diferentes frações do material particulado em suspensão apresentou um enriquecimento nas partículas mais finas, assim como na fração coloidal, evidenciando o tipo de transporte deste elemento para o oceano. ${ }^{14}$

O principal objetivo do presente estudo foi estabelecer a distribuição de mercúrio total nos sedimentos marinhos profundos (750-1.950 m de lâmina d'água) da Margem Continental da Bacia de Campos - RJ e suas associações com alguns suportes geoquímicos (ex. carbono orgânico, carbonato, $\mathrm{Fe}, \mathrm{Mn}, \mathrm{Al}$, fração silte e argila) visando o entendimento de mecanismos de acumulação de $\mathrm{Hg}$ em sedimentos marinhos profundos do Atlântico Sul.

\section{PARTE EXPERIMENTAL}

A Bacia de Campos situa-se na porção sudeste do Brasil em águas pertencentes ao Estado do Rio de Janeiro, sendo limitada ao 
sul pelo Arco de Cabo Frio e ao norte pela Bacia do Espírito Santo. Abrange uma área com cerca de 100.000 km² até a lâmina d'água de $3.000 \mathrm{~m}$. Esta região é a maior produtora nacional de petróleo em regiões marinhas, representando uma das mais importantes reservas petrolíferas oceânicas do Brasil. ${ }^{15} \mathrm{O}$ vento predominante na região é do quadrante NE, sendo a Bacia de Campos influenciada principalmente pela Corrente do Brasil (CB), caracterizando a circulação superficial da costa SE brasileira. O relevo da porção norte da bacia é estreito e íngreme, diferenciado da porção sul onde o talude é largo e com menor declive. ${ }^{16} \mathrm{~A}$ plataforma média da bacia é dominada por areia rica em feldspato e por carbonatos biogênicos. ${ }^{17}$

A área de estudo foi dividida entre região Norte e Sul, com 20 e 24 estações de coleta, respectivamente. As isóbatas de amostragem foram 750, 1050, 1350, 1650 e $1950 \mathrm{~m}$, selecionadas previamente pela PETROBRAS através do Projeto de Caracterização Ambiental de Águas Profundas da Bacia de Campos coordenado pelo CENPES/PETROBRAS. Duas campanhas foram realizadas, sendo a primeira em 2002 e a segunda em 2003 (C1-11/2002; C2-06/2003) ambas a bordo do navio rebocador Astro Garoupa.

Um box-corer da Ocean Instruments "Usnel Spade Corer", foi utilizado na amostragem das 44 estações das quais a fração de 0-2 $\mathrm{cm}$ de sedimentos superficiais foi a trabalhada neste estudo. Todas as amostras foram mantidas sob refrigeração no navio até o transporte para o laboratório. No laboratório os sedimentos foram separados por via úmida na sua fração $<2,0 \mathrm{~mm}$, secos em estufa $\left(40^{\circ} \mathrm{C} / \sim 48 \mathrm{~h}\right)$ e homogeneizados em moinho de bolas a $500 \mathrm{rpm}$ durante $10 \mathrm{~min}$.

$\mathrm{O}$ conteúdo de carbonato foi determinado por gravimetria em sedimento tratado com $\mathrm{HCl}(1 \mathrm{M}),(1 \mathrm{~g}: 10 \mathrm{~mL})$. Os valores foram então calculados a partir da diferença entre o peso inicial e final da amostra após o tratamento ácido. ${ }^{18}$ Os valores foram expressos em percentual (\%) e a precisão obtida com três repetições foi de $98 \%$. O carbono orgânico foi determinado em um analisador elementar CHNS/O Perkin Elmer (2.400 Series II), na sub-alíquota da amostra tratada para determinação de carbonatos. ${ }^{19}$ Os resultados de carbono orgânico foram posteriormente corrigidos considerando-se o conteúdo de carbonatos em cada amostra. As análises foram feitas em triplicata, apresentando uma precisão de $95 \%$.

A granulometria das amostras foi realizada de acordo com a metodologia proposta pela Associação Brasileira de Normas Técnicas adaptada para sedimentos marinhos (ABNT - NBR 7181). ${ }^{20}$ Aproximadamente $50 \mathrm{~g}$ de sedimentos foram mantidas em $125 \mathrm{~mL}$ da solução de hexametafosfato de sódio e carbonato de sódio durante 24 h. Em seguida, todo o sedimento foi transferido para o jogo de peneiras onde foi determinada a participação das diferentes frações da areia (Grossa, Média e Fina) e seus percentuais calculados. A fração silte e argila $(<63 \mu \mathrm{m})$ permaneceu em repouso na solução para desagregação e, posteriormente, foi transferida para uma proveta de $1000 \mathrm{~mL}$ e adicionou-se água destilada para completar o volume. Após, o material da proveta foi agitado por movimentos semicirculares durante $1 \mathrm{~min}$, para total desagregação das partículas. Em seguida o sedimento foi colocado em uma bancada nivelada e foram realizadas 12 leituras de densidade com intervalos previamente estabelecidos durante $24 \mathrm{~h}$. Em cada leitura mediu-se a temperatura do ar ambiente e os valores da fração silte e argila foram calculados a partir da lei de Stokes.

As determinações do $\mathrm{Al}, \mathrm{Fe}$ e $\mathrm{Mn}$ totais foram realizadas através de uma extração ácida de $\mathrm{HF}: \mathrm{HNO}_{3}$ (5:3) mantida a uma temperatura de $120^{\circ} \mathrm{C}$ por aproximadamente $12 \mathrm{~h}$, baseado em Kersten e Förstner. ${ }^{21} \mathrm{~A}$ fração reativa dos metais (Al, Fe e $\mathrm{Mn}$ ) foi obtida a partir da adição da solução ácida de $\mathrm{HCl} 1 \mathrm{M} \cdot{ }^{22}$ A determinação das concentrações de metais totais e reativos foi realizada utilizando-se o IPC-AES (Varian-Liberty Series II), trabalhando com os seguintes limites de detecção: Mn 0,1 $\mathrm{g} \mathrm{g}^{-1}$; Fe $0,2 \mu \mathrm{g} \mathrm{g}^{-1}$ e Al 0,2 $\mu \mathrm{g} \mathrm{g}^{-1}$. A exatidão calculada através de padrões certificados (NIST 2702 e 1646a) ficou acima de $93 \%$ e o coeficiente de variação analítico entre réplicas foi inferior a $10 \%$.

A extração do mercúrio total foi baseada na metodologia descrita por Bastos et al.. ${ }^{23} \mathrm{Em} \mathrm{1,0} \mathrm{g}$ de sedimento seco, em triplicata, adicionaram-se $2 \mathrm{~mL}$ de água Milli-Q e 5,0 $\mathrm{mL}$ de água régia $\left(\mathrm{HNO}_{3}+\mathrm{HCl}\right.$; 1:3). O material foi aquecido a $60{ }^{\circ} \mathrm{C} / 5 \mathrm{~min}$ em bloco digestor e após o resfriamento adicionaram-se mais 5,0 mL de água Milli-Q e $10 \mathrm{~mL}$ de solução de $\mathrm{KMnO}_{4}$ a 5\%, levando-se novamente ao bloco digestor a $60{ }^{\circ} \mathrm{C} / 30 \mathrm{~min}$. Novamente a solução foi resfriada e titulada com $\mathrm{NH}_{2} \mathrm{OH} . \mathrm{HCl}$ a $12 \%$. O extrato final foi filtrado em papel Whatman 40 e aferido até $25 \mathrm{~mL}$ com água Mili-Q. A leitura foi realizada pelo analisador de Hg, Quick Trace M-7500 da Cetac-Varian. A precisão ficou acima de $90 \%$ e a exatidão, calculada através de padrões certificados (NIST 2702 e 1646a), variou entre 85 e $90 \%$.

Para a análise estatística foram empregados os testes não paramétricos Mann-Withney para comparação entre as áreas Norte e Sul e o teste de Kruskal-Wallis para a comparação entre as diferentes isóbatas. Todos os cálculos foram realizados utilizando-se o programa Statistica (versão 5.5).

\section{RESULTADOS E DISCUSSÃO}

Os dados de granulometria mostram que a fração silte - argila foi predominante em todas as isóbatas, apresentando médias gerais de 72 e $79 \%$ para região norte e 78 e $81 \%$ para sul nas campanhas 1 e 2, respectivamente, seguida das frações de areia fina e média (Figuras 1a e 1b). O enriquecimento da fração silte - argila com o aumento da profundidade nas duas regiões é comum em áreas de turbidito. Esse padrão está associado ao transporte de material fino e de baixa densidade a longas distâncias, como descrito em outros trabalhos..$^{24,25}$

$\mathrm{O}$ teor de carbonato aumentou com a profundidade para as duas regiões em ambas as campanhas, sendo as maiores concentrações observadas na isóbata de 1950 m (Figura 2a). Cabe ressaltar, no entanto, que durante a primeira campanha este padrão de enriquecimento não foi muito evidente, pois os teores estiveram sempre próximos a $30 \%$ entre 750 e $1650 \mathrm{~m}$, com um aumento na isóbata de $1950 \mathrm{~m}$ como encontrado na segunda campanha $(\sim 45 \%)$. No entanto, comparandose as duas campanhas e as regiões Norte e Sul não foram observadas diferenças significativas para o carbonato $(\mathrm{p}>0,05)$. Abílio et al. ${ }^{26}$ encontraram um resultado similar para o carbonato em sedimentos da Bacia de Santos. Em adição, Carvalho et al. ${ }^{27}$ encontraram altos teores de carbonato, variando de 23 a 99\%, para sedimentos da região oceânica de Rio de Janeiro, utilizando o mesmo extrator ( $\mathrm{HCl} 1 \mathrm{M}$ ), através do projeto REVIZEE (Programa Recursos Vivos da Zona Econômica Exclusiva). De acordo com a autora, a abundância de carbonato é devida à elevada biomassa de algas calcárias associada a outros organismos que encontram, na plataforma continental norte e nordeste brasileira, condições favoráveis ao seu desenvolvimento.

O carbono orgânico apresentou uma distribuição com o aumento da profundidade apenas na região Norte da campanha 2, com exceção da isóbata de $1950 \mathrm{~m}$. As concentrações de carbono orgânico nas duas

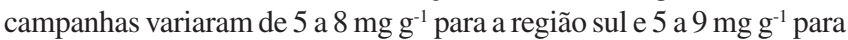
a região norte (Figura $2 b$ ). De acordo com estudos realizados na mesma região, as análises da composição isotópica do carbono e nitrogênio não mostraram diferenças espaciais significativas $(\mathrm{p}>0,05)$, tanto ao longo das isóbatas quanto no eixo Norte e $\mathrm{Sul}\left(\delta^{13} \mathrm{C}=-21,1 \pm 0,40 \%\right.$ 。 e $\delta^{15} \mathrm{~N}=7,10 \pm 1,00 \%$; $\delta^{13} \mathrm{C}=-21,1 \pm 0,50 \%$ o e $\delta^{15} \mathrm{~N}=7,70 \pm 1,50 \%$, respectivamente), ${ }^{28}$ corroborando com os resultados encontrados.

Em relação aos metais Al, Fe e Mn e a fração reativa comparandose as duas campanhas não foram observadas diferenças significativas entre as áreas norte e sul para nenhuma isóbata ( $p>0,05)$. A abundância absoluta dos metais (total) apresentou-se distribuída $\mathrm{Al}>\mathrm{Fe}>\mathrm{Mn}$ 
Campanha 1

(a)

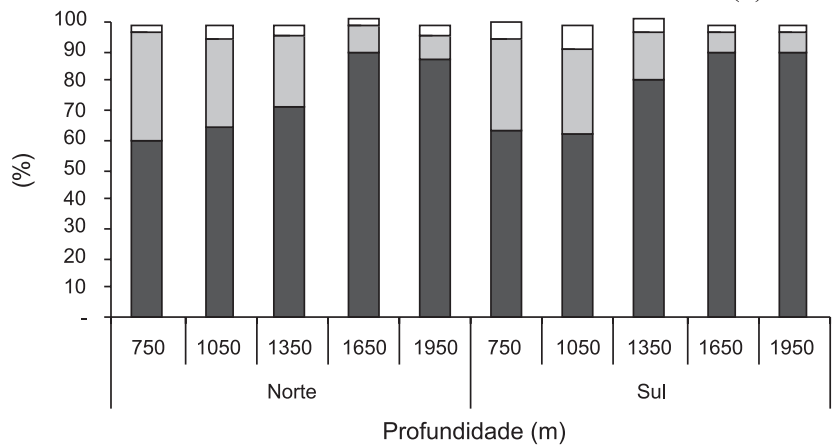

Silte + Argila $\square$ Areia Fina $\square$ Areia Média $\square$ Areia Grossa

Campanha 2

(b)

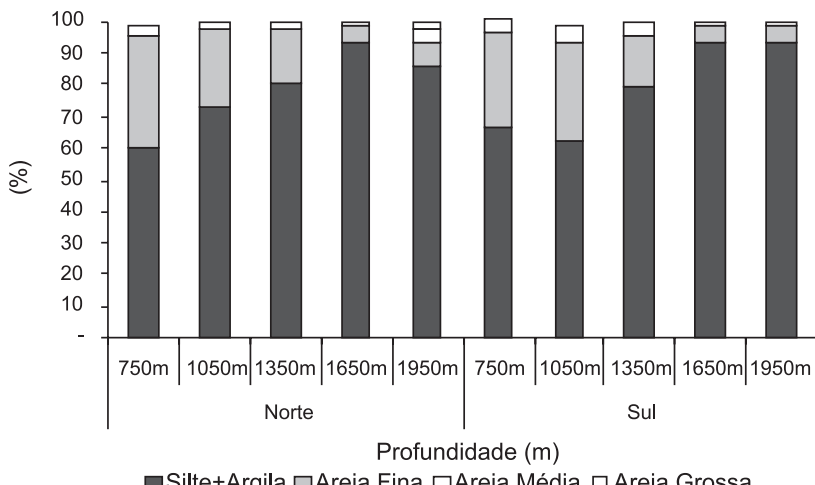

Figura 1. Granulometria das isóbatas para a área estudada: (a) campanha 1 e (b) campanha 2

para as duas campanhas. Entre as campanhas 1 e 2, houve um aumento das concentrações de $\mathrm{Al}$ para a região sul e uma diminuição para a região norte. $\mathrm{O} \mathrm{Mn}$ apresentou os maiores valores médios na campanha 1, enquanto o Fe obteve concentrações muito próximas entre as regiões norte e sul (campanhas 1 e 2) (Tabela 1). Os resultados encontrados estão acima dos valores observados na plataforma continental a cerca de $100 \mathrm{~m}$ de profundidade para a Bacia de Campos: $\mathrm{Al}\left(4,8 \mathrm{mg} \mathrm{g}^{-1}\right), \mathrm{Fe}\left(4,5 \mathrm{mg} \mathrm{g}^{-1}\right)$ e $\mathrm{Mn}\left(74 \mu \mathrm{g} \mathrm{g}^{-1}\right) .{ }^{12}$ Esta diferença nas concentrações destes elementos está relacionada ao nível de base regional e/ou à variação da granulometria, já que foi observado um maior teor de silte-argila com o aumento da profundidade..$^{29}$ Por outro lado, estes valores estão muito abaixo quando comparados à rocha sedimentar: Al (92 mg g $\left.{ }^{-1}\right), \mathrm{Fe}\left(47 \mathrm{mg} \mathrm{g}^{-1}\right)$ e Mn $\left(850 \mu \mathrm{g} \mathrm{g}^{-1}\right){ }^{1}$ Dessa forma, esses resultados parecem sugerir uma baixa contribuição do aporte continental, devido aos valores serem considerados baixos.

Todos os metais reativos apresentaram as maiores concentrações na região norte: $\mathrm{Al}\left(115\right.$ e $\left.168 \mu \mathrm{g} \mathrm{g}^{-1}\right)$, Fe $\left(263\right.$ e $\left.364 \mu \mathrm{g} \mathrm{g}^{-1}\right)$ e $\mathrm{Mn}$ (159 e $197 \mu \mathrm{g} \mathrm{g}^{-1}$ ) campanhas $1 \mathrm{e} 2$, respectivamente (Tabela 1). A presença de caniôns e as mudanças nos parâmetros físico-químicos na interface água-sedimento e água intersticial, além de interação com a biota que podem proporcionar a mudança de fase dos elementos aumentando sua reatividade no ambiente, podem justificar a diferença observada entre as regiões norte e sul. A fração reativa em $\mathrm{HCl} 1 \mathrm{M}$ extrai os metais que se encontram retidos na superfície das partículas sedimentares: nos sítios de troca catiônica, fracamente complexados por matéria orgânica, e adsorvidos e/ou coprecipitados por óxidos e hidróxidos de Fe e Mn e carbonatos. ${ }^{18}$ Logo, determinadas mudanças nas condições físico-químicas podem liberar metais fracamente ligados aos sedimentos de fundo para a coluna d'água, enquanto outras

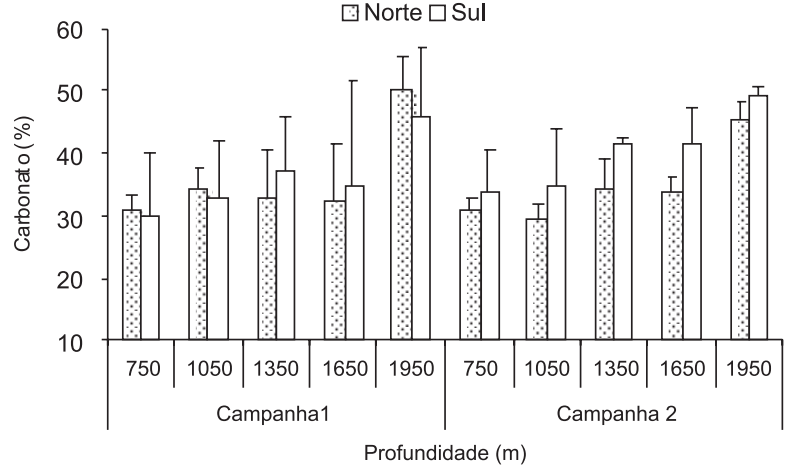

(b)

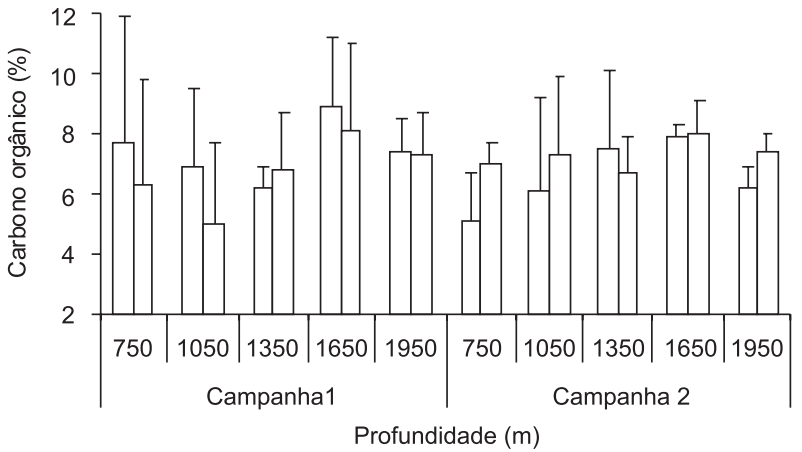

Figura 2. Concentrações médias de carbonato (a) e carbono orgânico (b) nos sedimentos da Bacia de Campos, RJ

podem favorecer a precipitação destes metais, o que pode explicar a variação observada no presente estudo.

Marinoni et al.,$^{30}$ utilizando a mesma metodologia do presente estudo ( $\mathrm{HCl} 1 \mathrm{M})$, estudaram a geoquímica e a composição mineralógica em sedimentos superficiais ricos em carbonato (30 a 90\%) na região oceânica SE do Chile (de 40 a 1000 m). Neste trabalho os autores analisaram vários elementos (ex. Fe, $\mathrm{Mn}, \mathrm{Sr}, \mathrm{Ca}$ e $\mathrm{Mg}$ ) e os baixos níveis de $\mathrm{Mn}$ e Fe na fase móvel (ex. extraído em $\mathrm{HCl} 1$ M) e apontaram para a possibilidade de uso destes elementos como indicadores do baixo grau de influência das fontes continentais na fase detrítica dos sedimentos.

As concentrações de $\mathrm{Hg}$-Total nos sedimentos estudados variaram de 8 a $26 \mathrm{ng} \mathrm{g}^{-1}$ (norte) e 8,3 a 29,6 $\mathrm{ng} \mathrm{g}^{-1}$ (Sul) na campanha 1 e de 11 a 35 (norte) e 8 a 29 (sul) na campanha 2 (Figura 3). Estes valores estão dentro das concentrações consideradas como nível de base para sedimentos da costa do sudeste do Brasil, os quais tipicamente variam de 10 a $50 \mathrm{ng} \mathrm{g}^{-1} .^{13-31}$

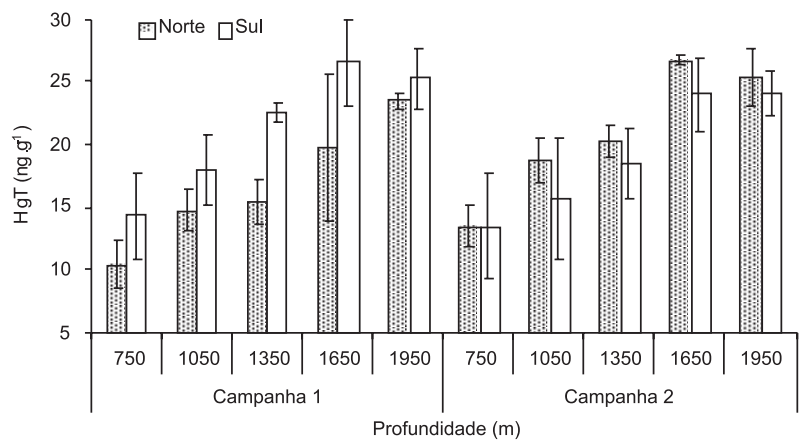

Figura 3. Concentrações médias de HgT por isóbata nos sedimentos da Bacia de Campos, RJ 
Tabela 1. Concentração média, mediana, máximo, mínimo, desvio padrão e coeficiente de variação para Fe, $\mathrm{Mn}$ e $\mathrm{Al}$ e suas frações reativas

\begin{tabular}{|c|c|c|c|c|c|c|c|}
\hline & & $\begin{array}{c}\mathrm{Al} \\
(\text { Total }) \\
\left(\mathrm{mg} \mathrm{g}^{-1}\right) \\
\end{array}$ & $\begin{array}{c}\mathrm{Fe} \\
(\text { Total }) \\
\left(\mathrm{mg} \mathrm{g}^{-1}\right) \\
\end{array}$ & $\begin{array}{c}\mathrm{Mn} \\
(\text { Total) } \\
\left(\mu \mathrm{g} \mathrm{g}^{-1}\right) \\
\end{array}$ & $\begin{array}{c}\mathrm{Al} \text { (Reativo) } \\
\left(\mu \mathrm{g} \mathrm{g}^{-1}\right)\end{array}$ & $\begin{array}{c}\text { Fe (Reativo) } \\
\left(\mu \mathrm{g} \mathrm{g}^{-1}\right) \\
\end{array}$ & $\begin{array}{c}\text { Mn (Reativo) } \\
\left(\mu \mathrm{g} \mathrm{g}^{-1}\right)\end{array}$ \\
\hline \multirow{14}{*}{ 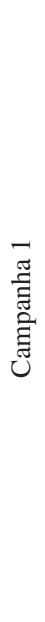 } & Norte & & & & & & \\
\hline & Média & 30 & 16 & 254 & 115 & 263 & 159 \\
\hline & Mediana & 27 & 14 & 250 & 117 & 268 & 151 \\
\hline & Máximo & 50 & 23 & 458 & 169 & 359 & 330 \\
\hline & Mínimo & 12 & 8 & 155 & 56 & 150 & 81 \\
\hline & DP & 14 & 6 & 83 & 31 & 53 & 55 \\
\hline & $\mathrm{CV}(\%)$ & 47 & 40 & 33 & 27 & 20 & 35 \\
\hline & Sul & & & & & & \\
\hline & Média & 22 & 17 & 298 & 102 & 227 & 142 \\
\hline & Mediana & 22 & 16 & 275 & 107 & 223 & 153 \\
\hline & Máximo & 42 & 28 & 945 & 138 & 330 & 229 \\
\hline & Mínimo & 12 & 10 & 1120 & 35 & 136 & 59 \\
\hline & DP & 7 & 5 & 166 & 27 & 47 & 51 \\
\hline & $\mathrm{CV}(\%)$ & 33 & 30 & 56 & 26 & 21 & 36 \\
\hline \multirow{14}{*}{ 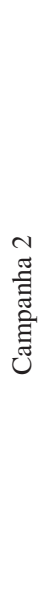 } & Norte & & & & & & \\
\hline & Média & 17 & 15 & 220 & 168 & 364 & 198 \\
\hline & Mediana & 15 & 15 & 217 & 159 & 366 & 191 \\
\hline & Máximo & 35 & 21 & 374 & 281 & 595 & 315 \\
\hline & Mínimo & 13 & 11 & 121 & 80 & 187 & 97 \\
\hline & DP & 5 & 3 & 63 & 56 & 110 & 60 \\
\hline & CV (\%) & 30 & 19 & 29 & 33 & 30 & 30 \\
\hline & Sul & & & & & & \\
\hline & Média & 26 & 16 & 218 & 138 & 268 & 175 \\
\hline & Mediana & 23 & 16 & 206 & 142 & 262 & 166 \\
\hline & Máximo & 45 & 26 & 407 & 203 & 396 & 314 \\
\hline & Mínimo & 8 & 9 & 121 & 70 & 149 & 70 \\
\hline & DP & 11 & 4 & 80 & 35 & 67 & 65 \\
\hline & $\mathrm{CV}(\%)$ & 43 & 27 & 36 & 25 & 25 & 37 \\
\hline
\end{tabular}

Estas variações, no entanto, despertam a necessidade de se iniciar uma abordagem mais ampla para a determinação adequada dos níveis de base regional para a Bacia de Campos, levando-se em consideração que a taxa de sedimentação é baixa neste ambiente e que a fração analisada foi de 0 a $2 \mathrm{~cm}$ de profundidade, de forma que as concentrações observadas refletem não só os processos atuais, mas integram variações que vêm ocorrendo na área de estudo a centenas de anos. Deve-se considerar que o espaço de tempo entre as duas coletas foi pequeno para causar um aporte de material natural capaz de promover alterações na qualidade dos sedimentos, o que pode explicar a baixa variação observada no presente estudo. Entretanto, caso variações expressivas fossem observadas, sugeririam um possível aporte antropogênico. As afirmações quanto à taxa de sedimentação foram realizadas considerando-se a taxa deposicional no campo Marlim leste da Bacia de Campos que foi estimada em 1,10 $\mathrm{cm} \mathrm{Ka}^{-1}$ (centímetros por cada mil anos) $^{32}$ e no talude da Bacia de Campos, onde a taxa variou de 1,04 a 1,81 $\mathrm{cm} \mathrm{Ka}^{-1}{ }^{33}$

Ao longo das isóbatas e nas duas campanhas para ambas as regiões foi observado um incremento das concentrações de $\mathrm{Hg}$-Total entre as

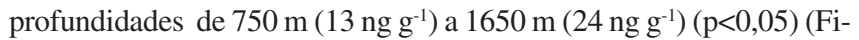
gura 3). Este padrão de aumento de $\mathrm{Hg}$ ao longo das isóbatas profundas reflete a distribuição granulométrica, onde ocorre um processo de acumulação natural de sedimentos finos com o aumento da profundidade. Trefry et $a l .{ }^{34}$ sugerem que concentrações mais elevadas observadas em sedimentos de isóbatas mais profundas se devem ao maior trajeto entre a superfície e os sedimentos, no qual as partículas vão capturando metais que estão disponíveis na água do mar.

Comparando-se as concentrações de Hg-Total entre as duas campanhas foi observada diferença significativa $(p<0,05)$ apenas entre os dados da área norte, com os maiores valores sendo observados na campanha 2. Já as concentrações na área sul foram semelhantes nas duas campanhas ( $\mathrm{p}>0,05)$. Fatores como morfologia do sedimento (ex: turbiditos e bioturbação) e diferenças no relevo entre as duas regiões podem ter influenciado neste resultado, já que esse é um ambiente de margem continental com presença de vários cânions (região norte) e platôs (região sul) que apresentam diferenças abruptas de declividade, gerando uma maior instabilidade para a região norte, onde uma migração pós-deposicional de sedimento tende a ocorrer com maior frequência, podendo gerar mudanças principalmente nas camadas superficiais.

A composição física dos sedimentos mostrou um enriquecimento significativo da fração silte-argila em direção a maiores profundidades, justificando o aumento das concentrações de Hg. Araújo et al., ${ }^{35}$ em teste onde se determinou $\mathrm{Hg}$ nos extratos de $\mathrm{HCl} 1 \mathrm{M}$, observaram que $82 \%$ de $\mathrm{Hg}$-Total se encontrava associado à fração reativa e apenas $18 \%$ na fração residual. Nesta fração reativa, o mercúrio pode ter como origem associações principalmente às fases trocáveis, redutíveis e carbonáticas como principal suporte geoquímico, uma vez que o carbono orgânico hidrolisado por esta solução é inferior a 5\%. ${ }^{36}$ 
A disponibilidade dos metais nos sedimentos depende de quão forte é a interação ligante/suporte. Através da análise de correlação é possível obter informações sobre a similaridade de fontes, bem como do comportamento ambiental dos metais. ${ }^{37}$ As correlações lineares simples do Hg-Total com suportes geoquímicos mostram uma estreita associação do Hg-Total com o tamanho das partículas ( $\mathrm{p}>0,01$ ), (Figura 4). Apesar da correlação entre o $\mathrm{Hg}$-Total e o Mn reativo não ter sido significativa, deve-se levar em consideração que cerca de 50 a $90 \%$ do $\mathrm{Mn}$ é reativo em $\mathrm{HCl} 1 \mathrm{M}$ (Tabela 1 ) podendo sugerir que este elemento atua como um importante suporte do $\mathrm{Hg}$ entre a fase dissolvida e sólida. Segundo Förstner e Wittman, ${ }^{1}$ os oxi-hidróxidos de Mn são carreadores importantes de numerosos elementos-traço influenciando, decisivamente, a química dos sedimentos marinhos durante os processos diagenéticos recentes. Além disso, Mn reativo geralmente serve como bom indicador das condições redox que predominam nos sedimentos. ${ }^{38}$ As demais correlações realizadas não foram significativas $(\mathrm{p}<0,01)$ para este estudo, a saber: $\mathrm{Hg}$-Total e Fe-Total; Hg-Total e Mn-Total; Hg-Total e Al-Total e Hg-Total e Fe reativo; $\mathrm{Hg}$-Total e $\mathrm{Mn}$ reativo; $\mathrm{Hg}$-Total e $\mathrm{Al}$ reativo; $\mathrm{Hg}$-Total e carbonato e Hg-Total e Corg.

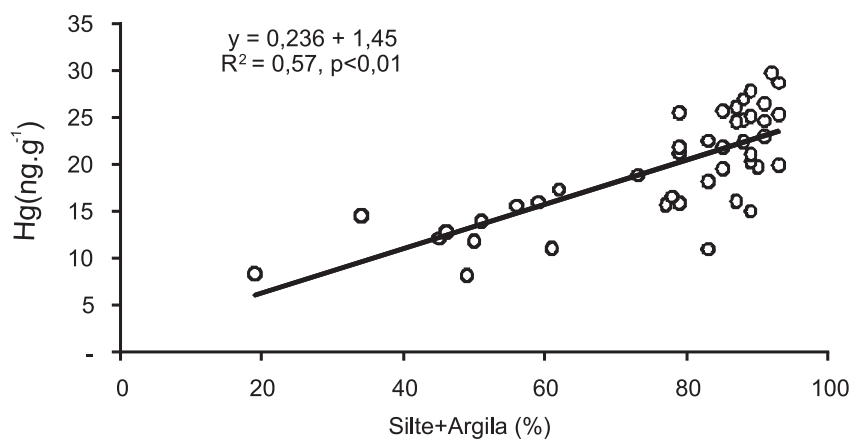

Figura 4. Correlação entre o $\mathrm{Hg}$ e a fração silte-argila nos sedimentos profundos da Bacia de Campos

Um dos aspectos que devem ser considerados nas abordagens ambientais é que o papel dos suportes geoquímicos pode mudar de importância dependendo das condições de meio, ou seja, força iônica, quantidade e qualidade da matéria orgânica e condições redox. Todas estas condições influenciam a estabilidade e o equilíbrio termodinâmico dos elementos em uma determinada condição ambiental. Assim, alguns exemplos podem ser apresentados quanto às mudanças das associações com os suportes geoquímicos em diferentes condições ambientais. Na Plataforma Continental da Bacia de Campos e na região costeira, um ambiente oxidante, Lacerda et al. ${ }^{39}$ observaram associações entre o Hg-Total e os teores de Corg, carbonato total, fração silte-argila e concentrações totais de $\mathrm{Al}$ e Fe, que demonstram a complexidade da geoquímica do $\mathrm{Hg} .{ }^{39}$ Em outro estudo realizado na Baía de Sepetiba foram encontradas correlações negativas do $\mathrm{Hg}$ com o carbono orgânico e o Mn-Total. ${ }^{31}$ Por outro lado, foi observada uma forte correlação com o Fe-Total, que foi confirmado como o principal suporte geoquímico para Baía de Sepetiba pela extração sequencial realizada no estudo.

O carbono orgânico e o Hg-Total apresentaram uma fraca correlação e este resultado indica uma refratabilidade da matéria orgânica presente neste pacote sedimentar. De acordo com Perin et al. ${ }^{40} \mathrm{a}$ matéria orgânica possui uma importância secundária na retenção/ complexação de metais em sedimentos salinos e oxidantes, comparada àquela exercida pelos óxidos e hidróxidos de $\mathrm{Mn} / \mathrm{Fe}$ e os sulfetos. Este comportamento pode estar ocorrendo nos sedimentos deste estudo, principalmente se considerarmos que o carbono orgânico presente nestas amostras demonstrou baixa reatividade em meio ácido e alcalino, sendo que a porção residual para ambos extratores foi de aproximadamente $95 \% .{ }^{36}$ Apesar da associação do carbonato com $\mathrm{Hg}$-Total não ter sido significativa, ainda é claro que na área de estudo este suporte geoquímico é importante, visto os teores observados (Figura 2a) seguem a mesma tendência da distribuição do $\mathrm{Hg}$, ou seja, enriquece com o aumento da profundidade.

O alumínio tem sido amplamente utilizado como elemento normalizador, por ser um elemento conservativo, proporcionando uma compensação sobre variações da granulometria, composição geoquímica, processos redox e sedimentação..$^{1-41}$ Possibilita, ainda, inferir sobre a origem natural dos metais nos sedimentos, quando as razões elementares entre fonte e destino forem próximas. ${ }^{42}$ Este elemento representa a quantidade de aluminossilicatos, a qual é a fase carreadora de metais com maior importância, devido a estar estreitamente representado pela fração silto-argilosa com composição geoquímica reativa e com importante papel nesse estudo.

A razão $\mathrm{Hg}$-Total/Al-residual foi realizada a partir da média dos pontos e utiliza o Al calculado pela diferença entre o Al-Total e o Al reativo. $\mathrm{O}$ uso de $\mathrm{Al}$ residual é mais satisfatório em comparação ao Al total, pois proporciona uma melhor estimativa da quantidade de aluminossilicatos, por apresentar características herdadas da matriz mineral sem a influência ao longo do transporte de fontes antropogênicas.$^{43} \mathrm{~A}$ razão do $\mathrm{Hg}$-Total/Al-residual foi maior em comparação às médias globais (crosta, sedimentos, folhelho médio e solos) ${ }^{6}$ (Tabela 2). A razão dos sedimentos do rio Paraíba do $\mathrm{Sul}^{44}$ foi mais elevada em comparação aos sedimentos da margem continental. De acordo com estes resultados pode-se inferir que existe uma diminuição da concentração de $\mathrm{Hg}$ na interface continente-oceano, e isto ocorre devido aos processos de sorção, com aparente predominância de dessorção, ou ainda através de processos de diluição (formação de coating) ocorridos durante o transporte e a sedimentação (Tabela 2).

Tabela 2. Comparação das taxas elementares dos sedimentos da margem continental da Bacia de Campos com MPS e sedimentos do rio Paraíba do Sul

\begin{tabular}{lc}
\hline Identificação & $(\mathrm{Hg} / \mathrm{Al}$ residual $) \times 10^{6}$ \\
\hline Sedimento Margem Continental & $1,13(0,32-4,22)$ \\
(Este estudo) & \\
Sedimento $^{44}$ & $3,32(0,12-8,1)$ \\
RPS & \\
Média Global $^{6}$ & 0,082 \\
Crosta Terrestre $^{6}$ & $0,20-0,35$ \\
Sedimentos & $0,28-0,30$ \\
Folhelho médio & $0,11-0,20$ \\
Solos &
\end{tabular}

Outro aspecto a ser considerado em relação ao transporte de metais na interface continente e oceano é o tamanho das partículas e reatividades dos coloides. Neste caso, em estudo realizado no rio Paraíba do Sul ficou caracterizado, entre os períodos de baixa e alta vazão, que o principal agente transportador é o material particulado em suspensão fino (MPS) $\left(0,1 \mu \mathrm{m}<\mathrm{MPS}_{\text {fino }}<0,63 \mu \mathrm{m}\right)$ variando de 68 a $96 \%$ da concentração de $\mathrm{Hg}$ na base volumétrica. No entanto, quando comparamos os resultados por massa de material separado em cada fração, observamos que a concentração de $\mathrm{Hg}$ na fração coloidal (FC) $(1 \mathrm{kD}<\mathrm{FC}<0,1 \mu \mathrm{m})$ é 5 vezes mais enriquecida em $\mathrm{Hg}\left(\sim 600 \mathrm{ng} \mathrm{g}^{-1}\right) .{ }^{14}$ Embora uma parcela deste material possa ser removida das águas na região estuarina, outra parcela poderá estar atingindo regiões mais distantes da costa. Esta evidência foi confirmada por Souza, ${ }^{45}$ através de isótopos de rádio, com um alcance de até $32 \mathrm{~km}$ da costa. No entanto, o Hg também chega aos sedimentos da margem continental pela deposição atmosférica e emissões do assoalho marinho, que são as principais fontes de $\mathrm{Hg}$ para esta área de estudo. 
Além disso, nossos resultados não suportam evidências para afirmar que as atividades petrolíferas estejam influenciando essas concentrações, já que também não existe um incremento significativo de $\mathrm{Hg}$ que justifique o enriquecimento por essas atividades.

Outros estudos realizados na Bacia de Campos encontraram concentrações de $\mathrm{Hg}$ menores que $70 \mathrm{ng} \mathrm{g}^{-1}$, em sedimentos coletados à profundidade de $900 \mathrm{~m},{ }^{46}$ entre $13 \mathrm{a} 80 \mathrm{ng} \mathrm{g}^{-1}$ para sedimentos da plataforma continental ${ }^{39}$ e de 145 a $30 \mathrm{ng} \mathrm{g}^{-1}$ no gradiente da pluma do rio à plataforma continental. ${ }^{13}$ Ogrinc et al.${ }^{47}$ encontraram altas concentrações de $\mathrm{Hg}$ em sedimentos profundos na bacia do Mar Mediterrâneo superiores às do presente estudo, entretanto deve-se ressaltar que esta área possui depósitos naturais e atividades vulcânicas e geotérmicas frequentes. Por outro lado, os valores encontrados em sedimentos da Antártica, onde segundo os autores a provável entrada de $\mathrm{Hg}$ se dá através de emissões vulcânicas regionais, ${ }^{48}$ estão dentro das concentrações observadas neste estudo (Tabela 3).

Tabela 3. Comparação entre as concentrações de Hg-Total nos sedimentos da área estudada com outros estudos

\begin{tabular}{|c|c|c|c|}
\hline Descrição & $\begin{array}{l}\text { Hg total } \\
\left(\mathrm{ng} \mathrm{g}^{-1}\right)\end{array}$ & $\begin{array}{l}\text { Profundidade } \\
\text { (m) }\end{array}$ & Comentários \\
\hline Bacia de Campos* & $10,4-26,8$ & $750-1950$ & Margem continental \\
\hline Bacia de Campos ${ }^{46}$ & $<70$ & 900 & Plataforma continental \\
\hline Bacia de Campos ${ }^{39}$ & $11,5-80$ & $95-110$ & Plataforma continental \\
\hline $\begin{array}{l}\text { Rio Paraíba do Sul - } \\
\text { Bacia de Campos }{ }^{13}\end{array}$ & $145-30$ & $10-1000$ & Pluma do rio - Estuário \\
\hline Rio Paraíba do Sul ${ }^{49}$ & $12,2-167$ & $0-0,01$ & $\begin{array}{c}\text { Bacia inferior - Itaocara a } \\
\text { Atafona }\end{array}$ \\
\hline Golfo do México ${ }^{34}$ & $48-558$ & - & $\begin{array}{l}\text { 100m dos campos de extra- } \\
\text { ção de petróleo }\end{array}$ \\
\hline Golfo do México ${ }^{34}$ & nov/92 & - & $\begin{array}{l}\text { Distante dos campos de } \\
\text { extração de petróleo }\end{array}$ \\
\hline Mar Mediterrâneo ${ }^{47}$ & $12-447$ & $910-4063$ & $\begin{array}{c}\text { Leste e Oeste da Bacia do } \\
\text { Mediterrâneo }\end{array}$ \\
\hline Antártica ${ }^{48}$ & $12 \pm 7$ & $5-300$ & Plataforma Continental \\
\hline
\end{tabular}

* Este estudo

\section{CONCLUSÕES}

$\mathrm{O}$ enriquecimento do $\mathrm{Hg}$ foi observado com o aumento da profundidade, demonstrando a importância da fração silte-argila, já que também existe um aumento dos sedimentos de granulometria fina nas isóbatas mais profundas. Não houve diferença entre as duas campanhas, demonstrando que não existem variações temporal e espacial dentro da nossa escala de observação. As concentrações de Hg-Total nesta área de estudo estão abaixo dos valores descritos para a Plataforma Continental adjacente $\left(\sim 40 \mathrm{ng} \mathrm{g}^{-1}\right)$, sendo consideradas baixas. A deposição atmosférica e as emissões do assoalho marinho podem ser consideradas importantes fontes de $\mathrm{Hg}$ para a área de estudo.

Outro aspecto que destacamos em relação ao transporte do $\mathrm{Hg}$ é a participação da fração coloidal, que desempenha um importante papel na dinâmica do Hg-Total na margem continental. Neste sentido, cresce a necessidade de ampliarmos o conhecimento da geoquímica do $\mathrm{Hg}$ nesta fração presente nas águas costeiras e oceânicas, assim como nos processos de transporte horizontal e vertical entre plataforma interna - margem continental - oceano profundo.

\section{MATERIAL SUPLEMENTAR}

Está disponível em http://quimicanova.sbq.org.br, na forma de arquivo .PDF, com acesso livre.

\section{AGRADECIMENTOS}

Ao técnico A. A. R. Gobo pelo tratamento inicial das amostras, ao Laboratório de Ciências Ambientais da UENF e à Petrobras pela possibilidade de coleta, análise do material e apoio financeiro e à tripulação do navio Astro Garoupa pelo apoio logístico na coleta das amostras. C. E. de Rezende é membro do Instituto Nacional de Ciência e Tecnologia que estuda a Transferência de Materiais na Interface Continente-Oceano (Proc.573.601/2008-9) e recebe apoio financeiro do CNPq (306.234/2007-6) e FAPERJ (E-23/102.697/2008).

\section{REFERÊNCIAS}

1. Förstner, U. E.; Wittman, G. T. W.; Metal Pollution In The Aquatic Environmental, $2^{\text {nd }}$ ed., Springer-Verlag: Berlin, 1983.

2. Mountouris, A.; Voutsas, E.;Tassios, D. ; Mar. Pollut. Bull. 2002, 44, 1136.

3. Azevedo F. A.; Toxicologia do Mercúrio, $1^{\mathrm{a}}$ ed., Rima: São Carlos, 2003.

4. Mason, R. P.; Sullivan, K. A.; Water Res. 1998, 32, 321.

5. Marins, R. V.; Paula Filho, F. J.; Lacerda, L. D.; Rodrigues, S. R.; Marques, W. S.; Quim. Nova 2004, 27, 763.

6. Salomons, W.; Förstner, U.; Metals in Hydrocycle, Springer-Verlag: New York, 1984.

7. Clarkson,T. W.; Crit. Rev. Clin. Lab. Sci. 1997, 34, 369.

8. Förstner, U.; Ahlf, W.; Calmano, W.; Water Sci. Technol. 1993, 28, 307.

9. Hedges, J. I.; Keil, R. G.; Mar. Chem. 1995, 49, 81.

10. Lechler, P. J.; Miller, J. R.; Lacerda, L. D.; Vinson, D.; Bonzongo, J. C.; Lyons, W. B.; Warwick, J. J.; Sci Total Environ. 2000, 260, 87.

11. Bisinoti, M. C.; Jardim, W. F.; Quim. Nova 2004, 27, 593.

12. Rezende, C. E.; Lacerda, L. D.; Ovalle, A. R. C.; Souza, C. M. M.; Gobo, A. A. R.; Santos, D. O.; Mar. Pollut. Bull. 2002, 44, 680.

13. Lacerda, L. D.; Carvalho, C. E. V.; Rezende, C. E.; Pfeiffer, W. C.; Mar. Pollut. Bull. 1993, 26, 220.

14. Almeida, M. G.; Rezende, C. E.; Souza, C. M. M.; Geochim. Bras. 2007, $21,111$.

15. Milani, E. J.; Brandão, J. A. S.; Zalán, L. P. V.; Gamboa, L. A. P.; Rev. Bras. Geof. 2000, 18, 3.

16. Habtec Engenharia Ambiental/Petrobras; Relatório de Impacto Ambiental, Bacia de Campos - Fpso P-50 Atividade de Produção e Escoamento de Petróleo e Gás Natural - Campo de Albacora Leste, Eia/ Rima: Rio de Janeiro, 2002.

17. Mello e Sousa, S. H.; Passos, R. F.; Fukumoto, M.; Silveira, I. C. A.; Figueira, R. C. L.; Koutsoukos, E. A. M.; Mahiques, M. M.; Rezende, C. E.; Marine Micropaleontology. 2006, 61, 40.

18. Leventhal, J.; Taylor, C.; Geochim. Cosmochim. Acta 1990, 54, 2621.

19. Skoog, D. A.; Leary, J. L.; Principles of instrumental analysis, $4^{\text {th }}$ ed., Harcounnt Brave Publishers: Orlando, 1992.

20. ABNT; NBR 7181 - Método de Ensaio para Análise Granulométrica em Solos, 1984.

21. Kersten, M., Förstner, U. Em Trace Element Speciation: Analytical Methods and Problems; Batley, G.E., ed.; CRC Press: Boca Raton, 1990.

22. Allen, H. E.; Fu,G.; Deng, B.; Environ. Toxicol. Chem. 1993, 17, 1441.

23. Bastos, W. R.; Malm, O.; Pfeiffer, W. C.; Cleary, D.; Cienc. Cult. 1998, $50,255$.

24. Di Leonardo, R.; Tranchida, G.; Bellanca, A.; Neri, R.; Angelone, M.; Mazzola, S.; Chemosphere 2006, 65, 2366.

25. Kontas, A.; J. Marine Systems 2006, 61, 67.

26. Abílio, G. S.; Cupelo, A. C. G.; Rezende, C. E.; Geochim. Bras. 2006, 20,71 .

27. Carvalho, A. M.; Pessoa Neto, A. R.; Santos, V. L. C. S.; Rev. Virtual 2005, 1,70 .

28. Rezende, C. E.; Ovalle, A. R. C.; Aragon, G. T.; Gobo, A. A. R.; Gobo, R. R.; Relatório técnico. Química de sedimentos: Distribuição espacial 
e temporal de matéria orgânica, carbonatos, carbono orgânico e inorgânico, nitrogênio total e concentração total de elementos maiores, menores e traços em sedimentos de fundo, 2006.

29. Siqueira, V. C.; Dissertação de Mestrado, Universidade Estadual do Norte Fluminense, Brasil, 2006.

30. Marinoni, L.; Setti, M.; Gauthier-Lafaye, F.; J. South Am. Earth Sci. 2000, 13,775 .

31. Marins, R. V.; Lacerda, L. D.; Paraquetti, H. H. M.; Paiva, E. C; Villas Boas, R. C. ; Environ. Contam. Toxicol. 1998, 61, 57.

32. Oliveira, D. H., Mello, R. M., Barreto, A. M. F., Strohschoen Jr, O.; Estudos Geológicos 2007, 17, 2.

33. Vicalvi, M. A.; Boletim de Geociências da PETROBRAS 1997, 11, 132.

34. Trefry, J. H.; Trocine, R. P.; McElvaine, M. L.; Rember, R. D.; Hawkins, L. T.; Environ. Geol. 2007, 53, 375.

35. Araújo, B. F.; Almeida, M. G.; Rezende, C. E.; trabalho não publicado.

36. Rezende, C. E.; Ovalle, A. R. C.; Salomão, M. S. B. M.; trabalho não publicado.

37. Jesus H. C.; Costa, E. A.; Mendonça, A. S. F.; Zandonade, E.; Quim. Nova 2004, 27, 378

38. Gagnon, C.; Pelletier, E.; Mucci,A. ; Mar. Chem. 1997, 59,159.
39. Lacerda, L. D.; Rezende, C. E.; Ovalle, A. R. C.; Carvalho, C. E. V.; Bull. Environ. Contam. Toxicol. 2004, 72, 178.

40. Perin, G.; Fabris, R.; Manente, S.; Wagener, A. R.; Hamacher, C.; Scotto, S.; Water Res. 1997, 31, 3017.

41. Aloupi, M.; Angelidis, M. O.; Environ. Pollut. 2001, 113, 211.

42. Roulet, M.; Lucotte, M.; Canuel, R.; Farella, N.; Courcelles, M.; Guimarães, J.; Mergler, D.; Amorim, M. ; Chem. Geol. 2000, 165, 243.

43. Ravichandran, M. ; Baskaran, M. ; Santschi, P. H. ; Bianchi, T. S. ; Environ. Sci. Technol. 1995, 29, 1495.

44. Araújo, B. F.; Almeida, M. G.; Rezende, C. E.; trabalho não publicado.

45. Souza, T. A.; Dissertação de Mestrado, Universidade Federal Fluminense, Brasil, 2008.

46. Pozebon, D.; Lima, E. C.; Maia, S. M.; Fachel, J. M. G.; Fuel 2005, 84, 53.

47. Ogrinc, N.; Monperrus, M.; Kotnik, J.; Fajon, V. K.; Vidimova, B.; Amouroux , D. ; Kocman, D.; Tessier, E.; Žižek, S.; Horvat, M.; Mar. Chem. 2007, 107, 31.

48. Bargagli, R.; Monaci, F.; Sanchez-Hernandez, J. C.; Cateni, D.; Mar. Ecol. Prog. Ser. 1998, 169, 65.

49. Almeida, M. G.; Souza, C. M. M.; Geochim. Bras. 2008, 22, 140. 


\section{DISTRIBUIÇÃO DE Hg TOTAL E SUAS ASSOCIAÇÕES COM DIFERENTES SUPORTES GEOQUÍMICOS EM SEDIMENTOS MARINHOS DA MARGEM CONTINENTAL BRASILEIRA: BACIA DE CAMPOS - RIO DE JANEIRO}

Beatriz Ferreira Araujo*, Marcelo Gomes de Almeida, Marcos Sarmet Moreira Barros Salomão, Renato Rosa Gobo, Verônica Caldas Siqueira, Álvaro Ramon Coelho Ovalle e Carlos Eduardo de Rezende

Laboratório de Ciências Ambientais, Centro de Biociências e Biotecnologia Universidade Estadual do Norte Fluminense, 28013-600 Campos dos Goytacazes - RJ, Brasil

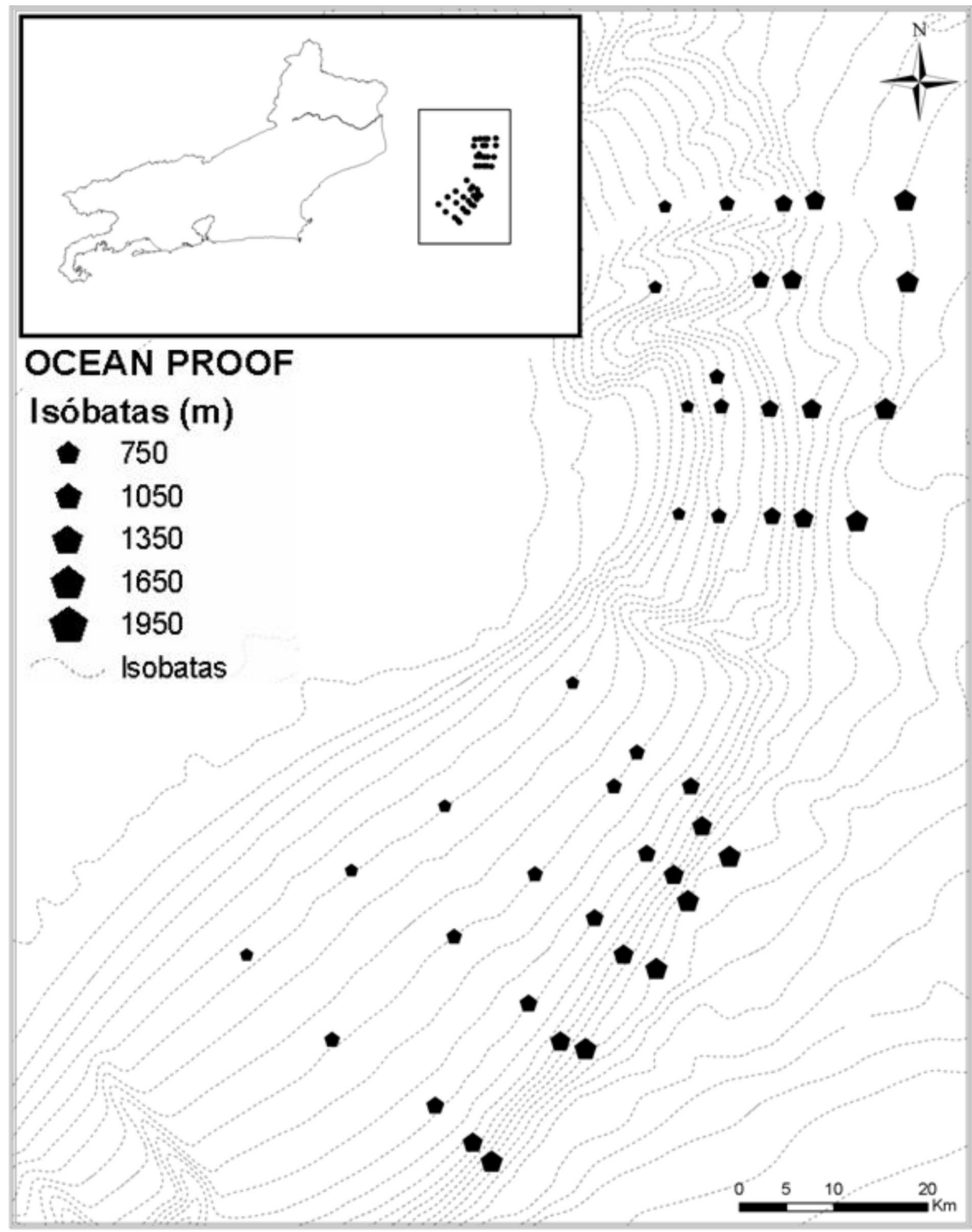

Figura 1S. Mapa do Estado do Rio de Janeiro, com indicação da Bacia de Campos e dos pontos de coleta. Fonte: Petrobrás 